\title{
Suppression of feline coronavirus replication in vitro by cyclosporin $A$
}

\author{
Yoshikazu Tanaka ${ }^{1 *}$, Yuka Sato ${ }^{1}$, Shuichi Osawa ${ }^{1}$, Mai Inoue ${ }^{1}$, Satoka Tanaka ${ }^{1}$ and Takashi Sasaki ${ }^{2}$
}

\begin{abstract}
The feline infectious peritonitis virus (FIPV) is a member of the feline coronavirus family that causes FIP, which is incurable and fatal in cats. Cyclosporin A (CSA), an immunosuppressive agent that targets the nuclear factor pathway of activated T-cells (NF-AT) to bind cellular cyclophilins (CyP), dose-dependently inhibited FIPV replication in vitro. FK506 (an immunosuppressor of the pathway that binds cellular FK506-binding protein (FKBP) but not CyP) did not affect FIPV replication. Neither cell growth nor viability changed in the presence of either CsA or FK506, and these factors did not affect the NF-AT pathway in fcwf-4 cells. Therefore, CSA does not seem to exert inhibitory effects via the NF-AT pathway. In conclusion, CSA inhibited FIPV replication in vitro and further studies are needed to verify the practical value of CSA as an anti-FIPV treatment in vivo.
\end{abstract}

\section{Introduction}

Coronaviruses are single-stranded RNA viruses that generally cause respiratory or intestinal infections such as severe acute respiratory syndrome (SARS) in humans and transmissible gastroenteritis (TGE) in pigs. Feline coronaviruses (FCoV) have been classified into two biotypes, comprising the ubiquitous feline enteric coronavirus (FECV) and infectious peritonitis virus (FIPV). The widely accepted theory in vitro is that FIPV arises by mutation of parental FECV in the gastrointestinal tract of infected cats, then systemically spreads and causes FIP that is fatal in cats [1,2]. Although the mutation sites are not fully understood, some accessory genes are candidates for the site of the critical mutations responsible for FIP [3,4]. Approximately $80 \%$ of all purebred cats are infected with feline coronavirus and among these, 5-12\% develop the classical symptoms of effusive/ wet FIP, the non-effusive/dry form of FIP, or a combination of the two [5].

Many strategies have been developed to cure FIP. Interferon $\omega$ inhibits FIPV in vitro but is ineffective in vivo [6]. Various other immunosuppressants, such as glucocorticoids and cyclophosphamide, have been studied, but although these drugs prolong life, the outcome of FIPV infection remains fatal [7]. Thus, an effective vaccine and therapeutic medicine against FIPV are still needed.

\footnotetext{
* Correspondence: ytanaka@nvlu.ac.jp

${ }^{1}$ Department of Veterinary Hygiene, Veterinary School, Nippon Veterinary and Life Science University, 1-7-1 Kyounan, Musashino, Tokyo, 180-8602, Japan Full list of author information is available at the end of the article
}

Investigators have reported that the immunosuppressant cyclosporin A (CsA) can suppress the genomic replication and transcription of several viruses [8-11]. Cyclophilins $(\mathrm{CyP})$ are cellular factors with high affinity for CsA [12] and comprise a family of cellular peptidyl-prolyl cis-trans isomerases (PPIases) that catalyze the cis-trans interconversion of the amino-terminal of peptide bonds to proline residues, thus facilitating changes in protein conformation as a chaperone protein [13]. Cyclosporin A blocks both the enzymatic activities of $\mathrm{CyP}$ that lead to the calcineurin $(\mathrm{CN})$ NF-AT and P-glycoprotein pathways. The capsid protein of human immunodeficiency virus type 1 (HIV-1) possesses a cyclophilin A (CyPA) binding site for incorporation into the virion $[14,15]$. A CsA-induced reduction in CyPA inhibits transport of the reverse-transcribed viral genome to the nucleus [16]. Cyclophilin B (CyPB) is another target of CsA that promotes hepatitis $\mathrm{C}$ virus $(\mathrm{HCV})$ replication by regulating the RNA-binding ability of the HCV NS5B protein. In addition, CyPA facilitates mouse cytomegalovirus (MCMV) replication and $\mathrm{CyPB}$ is required for the infectious entry of human papillomavirus type 16 .

Here, we show that CsA inhibits intracellular replication of the FIPV genome and viral protein expression in vitro independently of the NF-AT pathway.

\section{Materials and methods Cell culture and virus \\ Felis catus whole fetus-4 (fcwf-4; American Type Culture Collection, VA, USA) cells were maintained in Dulbecco's}


modified Eagle's medium (D-MEM, Sigma-Aldrich, Tokyo, Japan) supplemented with $10 \%$ fetal bovine serum (JRH, Nissui, Tokyo, Japan). We propagated FIPV (79-1146 strain; a gift from Dr Tsutomu Hodatsu, Kitasato University, Japan) in fcwf- 4 cells and then purified them by linear sucrose gradient ultracentrifugation.

Cells treated with cyclosporin A, cyclosporin H, and FK506 We inoculated fcwf-4 cells with FIPV 79-1146 at a multiplicity of infection (MOI) of 1 plaque-forming unit (pfu) per cell to study their effects on FIPV infection. After adsorption for $1 \mathrm{~h}$ at $37^{\circ} \mathrm{C}$, the medium containing the virus was removed, and the cells were rinsed three times with phosphate-buffered saline [PBS (-)] and incubated with or without various concentrations of CsA (Sigma-Aldrich), cyclosporin H (CsH; Cosmobio, Tokyo, Japan) and FK506 (Sigma-Aldrich) for $20 \mathrm{~h}$. The cells were then processed for photography. The cells were adsorbed with FIPV, rinsed three times with PBS (-), overlaid with DMEM containing $5 \%$ fetal bovine serum and $2 \%$ Agarose S (Nippon Gene, Toyama, Japan). After a 20-h incubation, the cells were stained with Giemsa solution and the plaques were counted.

\section{Plasmid constructs}

We isolated the nucleocapsid $(\mathrm{N})$ gene of FIPV from the plasmid pBRDI1 (provided by Dr Peter J. M. Rottier, Institute of Biomembranes, Utrecht University, The Netherlands) containing the FIPV 79-1146 genome, using the polymerase chain reaction (PCR) with the primers 5'-ACAAGGAC GACGACGACAAGGCCACACAGGGACAACGCG-3' and 5'-CCGGAATTCTTAGTTCGTAACCTCATCAA-3' for the first amplification. The products of the first amplification were purified by electrophoresis and gel extraction, and then a second PCR proceeded with the purified products and primers containing a FLAG-tagged sequence: (5'GCCACCATGGACTACAAGGACGACGACGACAAG-3' and 5'-CCGGAATTCTTAGTTCGTAACCTCATCAA-3'). The second PCR products were cut with Nco I and Eco RI and subcloned into the sites between Nco I and Eco RI of the pTri-EX 1.1 vector (Novagen, Takara Bio, Kyoto, Japan) to express FLAG-tagged N protein.

\section{Real-time, quantitative reverse transcription-PCR (qRT-PCR)}

The fcwf-4 cells were infected at an MOI of 1 pfu per cell and then incubated with or without CsA, CsH or FK506. The medium was removed at $22 \mathrm{~h}$ post-infection, and RNAiso-plus (Takara Bio) was added to the cells for RNA preparation according to the manufacturer's protocol. Total RNA (800 ng) was reverse-transcribed using the PrimeScript RT-PCR kit (Perfect Real Time; Takara Bio). Viral cDNA were quantified by real-time PCR using the forward and reverse primers to the FIPV-N gene
(5'-TGGCCACACAGGGACAAC-3') and (5'-AGAAC GACCACGTCTTTTGGAA-3'), and the TaqMan probe (FAM-GTTGCA GCACAGCCAGCATAAACAA-BHQ-1). Reaction mixtures were prepared according to the manufacturer's protocol using Premix EXTaq (Takara Bio), and sequences were amplified using a 7500 Sequence Detection System (Applied Biosystems, Tokyo, Japan) under the following cycling conditions: initial denaturation at $95^{\circ} \mathrm{C}$ for $10 \mathrm{~s}$ and 40 cycles at $95^{\circ} \mathrm{C}$ for $5 \mathrm{~s}$ and $60^{\circ} \mathrm{C}$ for $36 \mathrm{~s}$ each. Complementary DNA to the FIPV-N gene was cloned into the pTri-EX 1.1 vector, which was serially diluted to provide standards for FIPV gene quantification. The viral RNA copy number was normalized using the feline $\beta$-2-microglobulin $(\beta 2 \mathrm{M})$ gene (GenBank; NM_001009876). The $\beta 2 M$ gene derived from fcwf-4 cells was cloned by PCR amplification using the following primers: f $32 \mathrm{M}-\mathrm{F}$ 5'-GGCGCGTTTTGTGGTCTTGGTC3' and f $32 \mathrm{M}-\mathrm{R}$ 5'-CACTTAACGACCTTGGGCTC-3'. The amplified PCR products were subcloned into pTAC-1 plasmids (BioDynamics Laboratory Inc. Tokyo, Japan) to provide standards for the $\beta 2 \mathrm{M}$ gene. We then quantified the feline $\beta 2 \mathrm{M}$ gene by real-time PCR using the forward (5'-CGCGTTTTGTGGTCTTGGTCTTGGT-3') and reverse (5'AAACCTGAACCTTTGGAGAATGC-3') primers for the $\beta 2 \mathrm{M}$ gene and detected the gene using the TaqMan probe, TAMRA-CGGACTGCTCTATCTGTC CCACCTGGA-BHQ-2.

\section{Luciferase assay}

Luciferase activities were quantified using pGL4.30 [luc2P/NFAT-RE/Hygro] (Promega, Tokyo, Japan), pRL-SV40 vectors for the NF-AT response assay and the interferon stimulation response was determined using the plasmid pISRE-TK hRluc (F) provided by RIKEN BioResource Center (Tsukuba, Japan) and the pGL3 promoter (Promega). Both reporter assays proceeded using the Dual-Luciferase Reporter Assay System (Promega). Briefly, the two reporter plasmids were co-transfected into fcwf-4 cells with or without CsA or FK506 for each assay. Recombinant feline IFN $\alpha$ (PBL Biomedical Laboratories, NJ, USA) was added at a concentration of 10 units $/ \mathrm{mL}$ to the culture medium to evaluate the response to interferon (IFN). Total cell lysates were prepared with Reporter Lysis buffer provided with the Dual-Luciferase Reporter Assay System at $48 \mathrm{~h}$ before the assay. Luciferase activities were quantified in triplicate assays using a Lumat LB9507 (Berthold Technologies, Tokyo, Japan).

\section{Western blotting}

The cell membranes were disrupted with cell lysis buffer [10 mM Tris- $\mathrm{HCl}, \mathrm{pH}$ 7.8, $1 \mathrm{mM}$ ethylenediamine tetraacetic acid (EDTA), 1 \% NP-40, $0.15 \mathrm{M} \mathrm{NaCl}$ including Complete Mini (Roche Diagnostics, Tokyo, Japan) at 

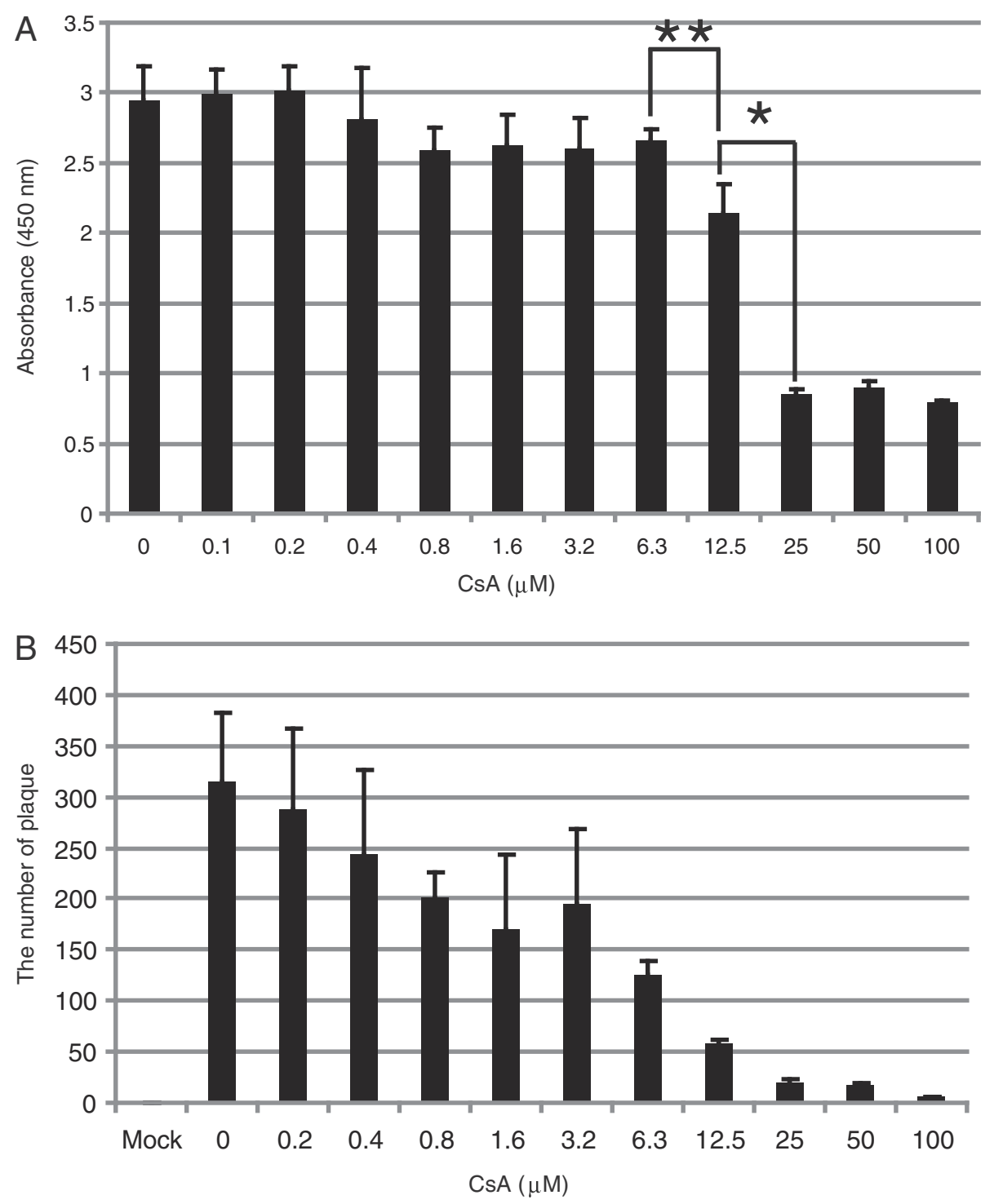

Figure 1 Cyclosporin A inhibits cytopathic effects of FIPV on fcwf-4 cells. (A) WST-8 assay of fcwf-4 cells cultured with indicated concentrations of CsA. Error bars indicate means \pm 2 SD. ${ }^{*} P,{ }^{* *} P<0.05$. (B) Plaques counted in FIPV-infected fcwf-4 cells incubated with or without CSA. Cells infected with FIPV were incubated with various concentrations of CSA.

$20 \mathrm{~h}$ after infection. The cell lysates were resolved by electrophoresis on 4-12\% Bis-Tris gels (Invitrogen, CA, USA) and Western blotted onto Immobilon-P membranes (Millipore, Tokyo, Japan). Non-specific protein binding was blocked with $5 \%$ non-fat dry milk for $1 \mathrm{~h}$ and then the membranes were incubated with anti-feline coronavirus nucleocapsid (N) antibody (FIPV3-70; MyBioSource, CA, USA) or anti- $\beta$-actin (Sigma-Aldrich) for $1 \mathrm{~h}$. Antigen signals were visualized by reacting proteins on the membranes with horseradish peroxidaseconjugated anti-mouse IgG antibody (Promega) followed by an enhanced chemiluminescence substrate (SuperSignal West Femto Maximum Sensitivity
Substrate; Thermo Scientific, Tokyo, Japan) according to the manufacturer's protocol.

\section{Cell viability assay}

We assayed WST-8 to evaluate cytotoxicity using the Cell Counting Kit-8 (Dojin Chemical Inc., Wako, Japan) according to the manufacturer's directions.

\section{Statistical analysis}

Statistical significance was determined using the Student's $t$ test. For all data analyzed, a significance threshold of $P<0.05$ was assumed. The values are expressed in some figures as means \pm standard deviation (SD). 

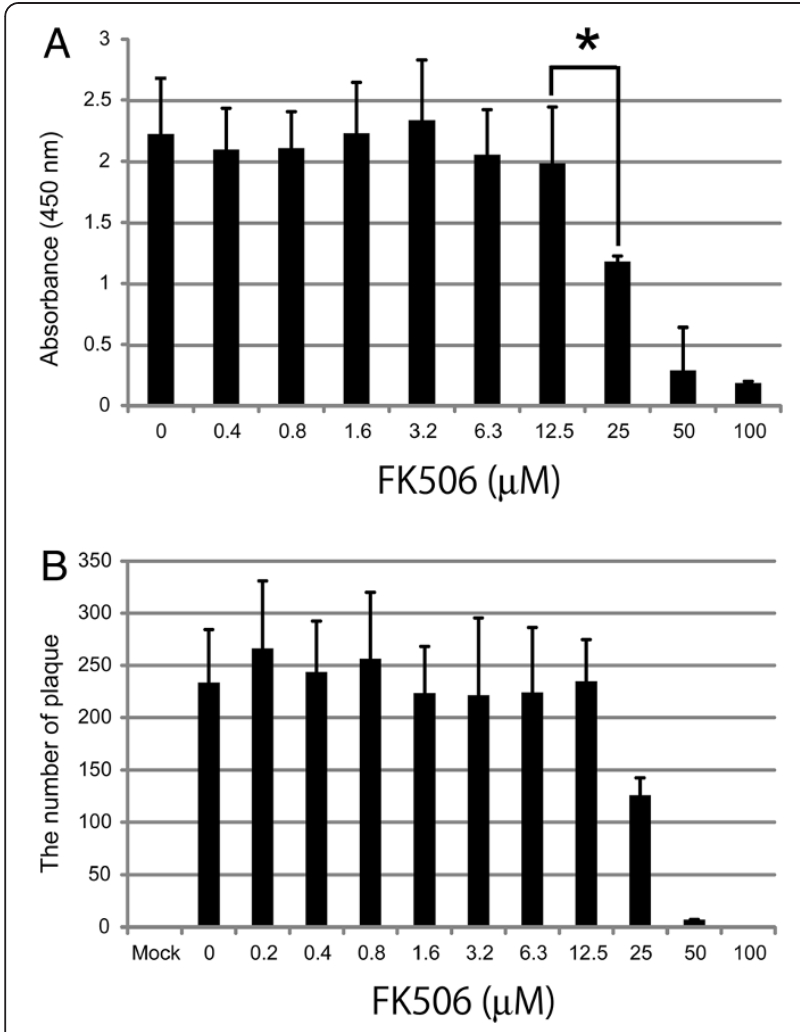

Figure 2 FK506 does not inhibit cytopathic effects of FIPV on fcwf-4 cells. (A) WST-8 assay of fcwf-4 cells cultured with various FK506 concentrations. Error bars indicate means \pm 2 SD. $* P<0.05$. (B) The numbers of plaque were counted in FIPV-infected fcwf- 4 cells incubated with or without FK506. Cells infected with FIPV were cultured with various concentrations of FK506.

\section{Results}

Inhibition of FIPV replication by CsA but not by FK506

We initially assessed the effects of CsA on FIPV RNA replication using cytotoxicity assays. Cyclosporin A at concentrations of $0-6.3 \mu \mathrm{M}$ (cytotoxic dose $\mathrm{s}_{50}$, $14.1 \pm 1.72 \mu \mathrm{M}$ ) did not affect fcwf-4 cell viability (Figure $1 \mathrm{~A}$ ) and dose-dependently reduced the numbers of FIPV plaques (Figure 1B), whereas $10 \mu \mathrm{M}$ CsA was slightly cytotoxic. Cyclosporin A blocks both the enzymatic activities of CyP that lead to the calcineurin (CN)-NF$\mathrm{AT}$ and the P-glycoprotein pathway. We therefore assessed the effect of various concentrations of FK506, which also blocks the NF-AT pathway, on cell viability to confirm that CsA inhibited FIPV through this pathway. The cytopathic inhibitory effects did not significantly differ in the presence or absence of $0.08-10 \mu \mathrm{M}$ FK506 (Figure 2B) and cell viability was not affected by $10 \mu \mathrm{M}$ FK506 (Figure 2A).

Quantitative RT-PCR showed that 0.63 - $10 \mu \mathrm{M}$ CsA dose-dependently suppressed FIPV RNA replication, whereas FK506 did not exert significant inhibitory effects, except at $10 \mu \mathrm{M}$ FK506 (approximately $30 \%$ reduction compared to $0 \mu \mathrm{M}$ FK506, $P<0.05$; Figure 3A). Western blotting showed that CsA, but not FK506 dosedependently decreased FIPV-N protein (Figure 3B).

We then examined whether the suppressive effects of CsA on FIPV replication depend on the inhibitory NFAT pathway or P-glycoprotein pathway by incubating FIPV-infected cells with $\mathrm{CsH}$, which specifically blocks the P-glycoprotein pathway. The results show that no inhibition occurred (data not shown).

\section{CsA does not elicit either an interferon-stimulated response or a NF-AT response in fcwf- 4 cells}

To determine whether the action of CsA and FK506 involves activation of interferon-stimulated gene responses in fcwf-4 cells, the ISRE-luciferase reporter plasmid, pISRETK hRluc (F) and pGL3 promoter plasmid as a normalization-control plasmid were transfected into fcwf-4 cells and cultured with feline interferon $\alpha$, CsA or FK506. The results of the dual-luciferase assay showed that none of these factors significantly affected luciferase activities at $48 \mathrm{~h}$ after transfection. These results indicate that fcwf-4 cells are unresponsive, even to interferon $\alpha$ (Figure 4A). Consequently, the action of CsA on intracellular FIPV replication does not involve the activation of interferonstimulated genes on fcwf-4 cells. Moreover, to evaluate the effects of CsA and FK506 on the calcineurin-NF-AT pathway in fcwf-4 cells, the NF-AT luciferase reporter plasmid, pGL4.30 [luc2P/NFAT-RE/Hygro] and pRL-SV40 as a normalization-control plasmid were transfected into fcwf4 cells that had been incubated with CsA or FK506. Neither CsA nor FK506 affected NF-AT luciferase activities in fcwf-4 cells (Figure 4B). These findings show that CsA does not influence the NF-AT pathway in fcwf-4 cells and that the inhibition of FIPV RNA replication by CsA is independent of the calcineurin NF-AT pathway.

\section{Discussion}

We discovered that CsA inhibits intracellular FIPV replication in vitro. The results of qRT-PCR and Western blotting showed that viral proliferation was strikingly inhibited at CsA concentrations between 0.16 and $10 \mu \mathrm{M}$. The results of cell viability assays showed that $10 \mu \mathrm{M}$ CsA was slightly cytotoxic. The inhibitory effects at this concentration should therefore be considered in the light of cytotoxicity. In contrast, immunosuppressive FK506 did not inhibit FIPV replication except at $10 \mu \mathrm{M}$ according to the qRT-PCR findings. However, the inhibitory effects of $0.08-10 \mu \mathrm{M}$ FK506 did not significantly differ on Western blots and the number of plaques did not significantly differ within the same concentration range of FK506. These findings might be related to the difference of the analytical detection sensitivity between qRT-PCR and Western blot assays. Cyclosporin A binds cyclophilins, whereas FK506 binds cellular FKBP. Each 

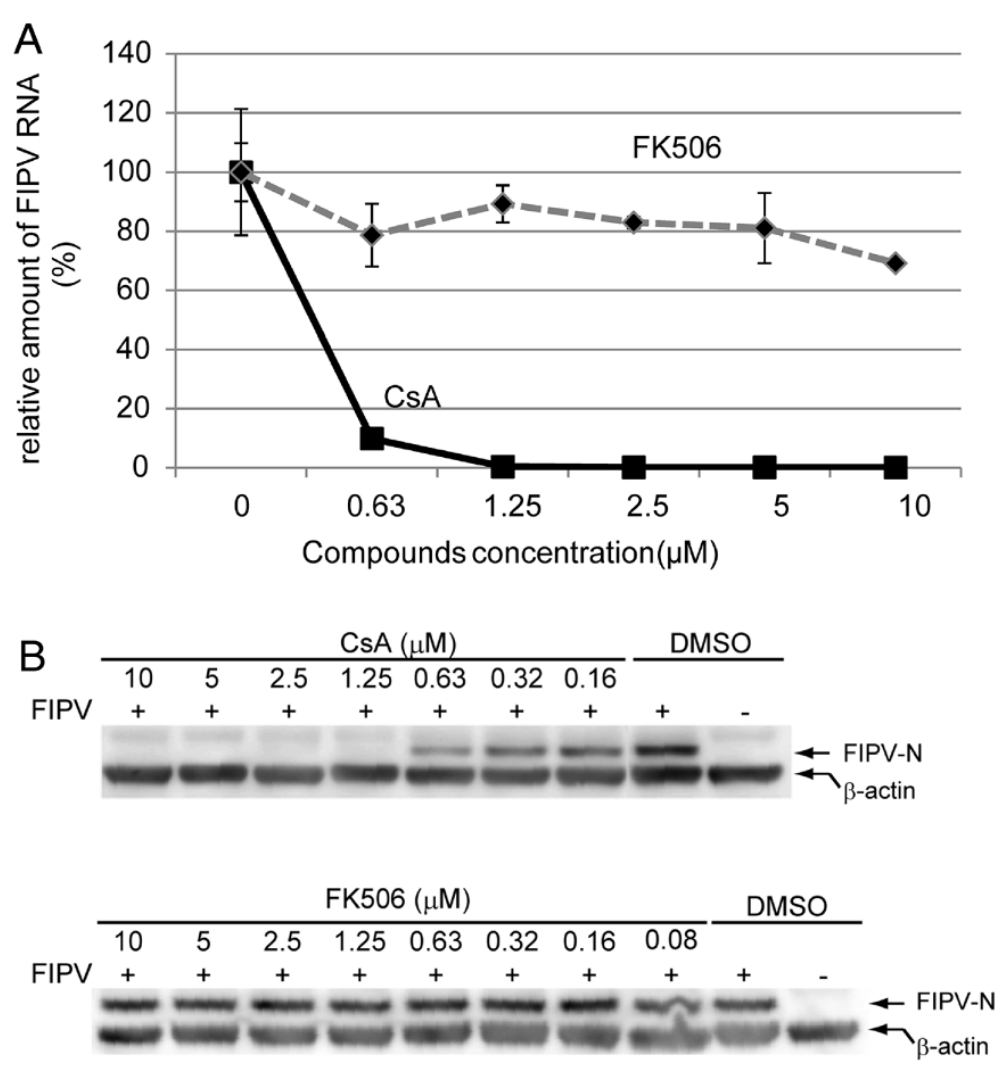

Figure 3 Cyclosporin A, but not FK506 suppresses FIPV replication in fcwf-4 cells. (A) Cells were infected with FIPV and incubated with or without the indicated concentrations of CSA or FK506. Total RNA extracted from cells $22 \mathrm{~h}$ later were reverse-transcribed to cDNA and assayed by real-time PCR assays targeting FIPV-N and $\beta 2 \mathrm{M}$ genes. Relative amounts of FIPV RNA expression data were normalized with $\beta 2 \mathrm{M}$ gene expression. Error bars indicate means \pm 2 SD. (B) Western blots of cells incubated with various concentrations of CSA and FK506. Lanes show total cellular proteins resolved by electrophoresis. Effects of CSA and FK506 were assessed using monoclonal anti-FIPV-N and anti- $\beta$-actin primary antibodies.

complex independently inhibits the phosphatase activity of calcineurin that mediates the NF-AT pathway, which is critical to the expression of cytokines and their receptors $[12,17]$. The results of NF-AT reporter assays indicate that neither CsA nor FK506 influenced NF-AT activities on fcwf-4 cells under our experimental conditions. Thus, the antiviral activity against FIPV is not involved in the suppression of gene responses regulated by NF-AT but instead is exerted through distinct mechanisms that are independent of FK506. Pfefferle et al. recently described that SARScoronavirus NSP1 overexpression increases signaling through the NF-AT pathway and enhances the induction of interleukin 2 [18]. Our data were somewhat inconsistent with these findings. The discrepancy might be explained in part by the fact that their experimental system included the addition of 13-O-Acetylphorbol 12-myristate (PMA) and ionomycin to the culture medium. Further knockdown studies of the NF-AT gene would clarify its role in FIPV proliferation. Furthermore, IFN $\alpha$ did not stimulate ISRE-promoter activities in fcwf-4 cells under these conditions. These data suggest that the action of CsA on the intracellular replication of FIPV is independent of the IFN pathway. This cell line might not be responsive to IFN $\alpha$ and would thus be useful for isolating feline coronavirus.

Cyclophilins have PPIase activity [13,19-21], contribute to the maturation of several proteins, and are involved in cell signaling, mitochondrial function (ATP synthesis), molecular chaperone activity, RNA splicing, stress response, gene expression, cholesterol transport, and the regulation of kinase activity [22-25]. Surface plasmon resonance technology and bioinformatics tools have found that the SARS coronavirus $\mathrm{N}$ protein binds to CyPA [26-28]. Therefore, the N protein of FIPV probably binds to $\mathrm{CyP}$ proteins to regulate viral replication. The roles of $\mathrm{CyP}$ in virus replication and the inhibitory effect by $\mathrm{CsA}$ on $\mathrm{HCV}$ and several other viruses have been studied [22-25]. The proposed mechanisms of the inhibitory effect of CsA mainly involve CypA and CypB in virus replication $[23,29,30]$. Based on these earlier findings, we believe that interaction between the FIPV genome is likely or that viral and CyP proteins play critical roles in viral replication and transcription. Further 


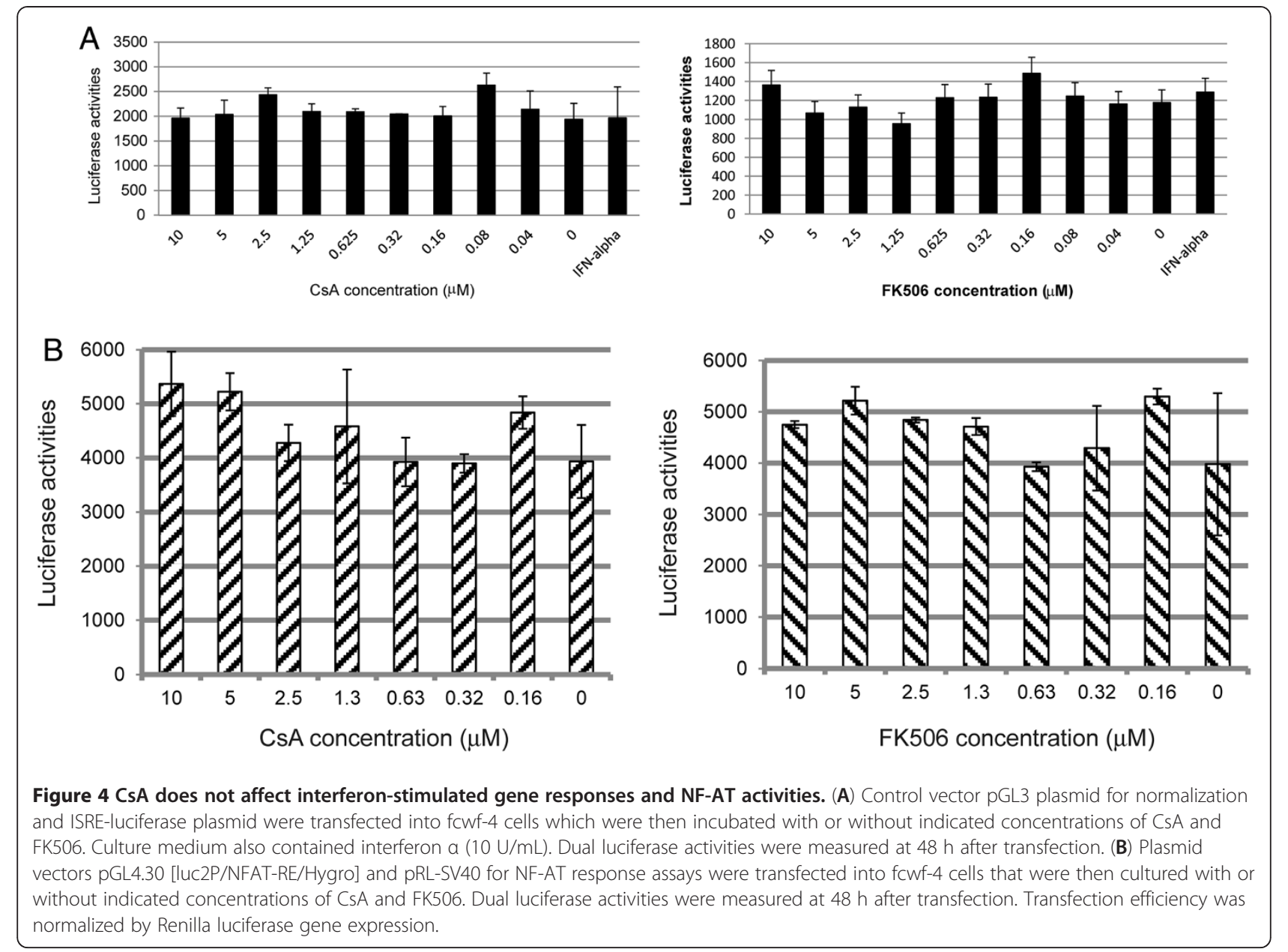

studies are required to resolve which $\mathrm{CyP}$ is critical for viral replication and which viral protein is required to form replication complexes with $\mathrm{CyP}$ and/or other cellular proteins.

Cats with clinically diagnosed FIP have very rarely been cured, although several therapeutic strategies have been attempted. Some cats treated with prednisolone and phenylalanine mustard or cyclophosphamide have gone into remission [26]. Various immunosuppressants, such as glucocorticoids and cyclophosphamide, may prolong life but do not alter the fatal outcome [4].

Further investigations using PPIase dominant-negative assays and RNA interference methods are warranted to clarify the role of CsA against PPIase in FIPV replication in vitro. In addition, whether CsA could be useful as FIP treatment in vivo remains to be determined

\section{Competing interests}

The authors declare that they have no competing interests.

\section{Acknowledgments}

This study was supported by a Grant-in-Aid for Scientific Research (C) from the Japan Society for the Promotion of Science and the Strategic Research Base
Development Program for Private Universities from the Ministry of Education, Culture, Sports, Science and Technology of Japan (MEXT), 2008-2012.

\section{Author details}

'Department of Veterinary Hygiene, Veterinary School, Nippon Veterinary and Life Science University, 1-7-1 Kyounan, Musashino, Tokyo, 180-8602, Japan.

${ }^{2}$ Department of Infection Control Science, Faculty of Medicine, Juntendo University, Tokyo, 113-8421, Japan.

\section{Authors' contributions}

YT carried out all experiments, participated in the data collection and analysis, and prepared the manuscript. SO, MI, and ST carried out cell culture and virus preparation. YS performed some of the biochemical experiments and TS carried out luciferase assays. All authors read and approved the final manuscript.

Received: 19 December 2011 Accepted: 30 April 2012

Published: 30 April 2012

\section{References}

1. Rottier PJ, Nakamura K, Schellen P, Volders H, Haijema BJ: Acquisition of macrophage tropism during the pathogenesis of feline infectious peritonitis is determined by mutations in the feline coronavirus spike protein. J Virol 2005, 79:14122-14130.

2. Pedersen NC, Boyle JF, Floyd K, Fudge A, Barker J: An enteric coronavirus infection of cats and its relationship to feline infectious peritonitis. Am J Vet Res 1981, 42:368-377. 
3. Kennedy M, Boedeker N, Gibbs P, Kania S: Deletions in the 7a ORF of feline coronavirus associated with an epidemic of feline infectious peritonitis. Vet Microbiol 2001, 81:227-234.

4. Pedersen NC: A review of feline infectious peritonitis virus infection: 1963-2008. J Feline Med Surg 2009, 11:225-258.

5. Berg AL, Ekman K, Belak S, Berg M: Cellular composition and interferongamma expression of the local inflammatory response in feline infectious peritonitis (FIP). Vet Microbiol 2005, 111:15-23.

6. Ritz S, Egberink H, Hartmann K: Effect of feline interferon-omega on the survival time and quality of life of cats with feline infectious peritonitis. $J$ Vet Intern Med 2007, 21:1193-1197.

7. Hartmann K, Ritz S: Treatment of cats with feline infectious peritonitis. Vet Immunol Immunopathol 2008, 123:172-175.

8. Billich A, Fricker G, Muller I, Donatsch P, Ettmayer P, Gstach H, Lehr P, Peichl P, Scholz D, Rosenwirth B: SDZ PRI 053, an orally bioavailable human immunodeficiency virus type 1 proteinase inhibitor containing the 2-aminobenzylstatine moiety. Antimicrob Agents Chemother 1995, 39:1406-1413.

9. Billich A, Hammerschmid F, Peichl P, Wenger R, Zenke G, Quesniaux V, Rosenwirth B: Mode of action of SDZ NIM 811, a nonimmunosuppressive cyclosporin $A$ analog with activity against human immunodeficiency virus (HIV) type 1: interference with HIV protein-cyclophilin A interactions. J Virol 1995, 69:2451-2461.

10. Bose $S$, Mathur M, Bates $P$, Joshi N, Banerjee AK: Requirement for cyclophilin A for the replication of vesicular stomatitis virus New Jersey serotype. J Gen Virol 2003, 84:1687-1699.

11. Watashi K, Ishii N, Hijikata M, Inoue D, Murata T, Miyanari Y, Shimotohno K: Cyclophilin B is a functional regulator of hepatitis C virus RNA polymerase. Mol Cell 2005, 19:111-122.

12. Handschumacher RE, Harding MW, Rice J, Drugge RJ, Speicher DW: Cyclophilin: a specific cytosolic binding protein for cyclosporin A. Science 1984, 226:544-547

13. Zydowsky LD, Etzkorn FA, Chang HY, Ferguson SB, Stolz LA, Ho SI, Walsh CT: Active site mutants of human cyclophilin A separate peptidyl-prolyl isomerase activity from cyclosporin A binding and calcineurin inhibition. Protein Sci 1992, 1:1092-1099.

14. Rosenwirth B, Oren DA, Arnold E, Kis ZL, Eggers HJ: SDZ 35-682, a new picornavirus capsid-binding agent with potent antiviral activity. Antiviral Res 1995, 26:65-82.

15. Rosenwirth B, Kis ZL, Eggers HJ: In vivo efficacy of SDZ 35-682, a new picornavirus capsid-binding agent. Antiviral Res 1995, 26:55-64.

16. Ptak RG, Gallay PA, Jochmans D, Halestrap AP, Ruegg UT, Pallansch LA, Bobardt MD, de Bethune MP, Neyts J, De Clercq E, Dumont JM, Scalfaro P, Besseghir K, Wenger RM, Rosenwirth B: Inhibition of human immunodeficiency virus type 1 replication in human cells by Debio-025, a novel cyclophilin binding agent. Antimicrob Agents Chemother 2008, 52:1302-1317.

17. Hait WN, Harding MW, Handschumacher RE: Calmodulin, cyclophilin, and cyclosporin A. Science 1986, 233:987-989.

18. Pfefferle S, Schopf J, Kogl M, Friedel CC, Muller MA, Carbajo-Lozoya J, Stellberger T, von Dall'Armi E, Herzog P, Kallies S, Niemeyer D, Ditt V, Kuri T, Züst R, Pumpor K, Hilgenfeld R, Schwarz F, Zimmer R, Steffen I, Weber F, Thiel V, Herrler G, Thiel HJ, Schwegmann-Wessels C, Pöhlmann S, Haas J, Drosten C, von Brunn A: The SARS-coronavirus-host interactome: identification of cyclophilins as target for pan-coronavirus inhibitors. PLOS Pathog 2011, 7:1002331.

19. Lodish HF, Kong N: Cyclosporin A inhibits an initial step in folding of transferrin within the endoplasmic reticulum. J Biol Chem 1991, 266:14835-14838.

20. Swanson SK, Born T, Zydowsky LD, Cho H, Chang HY, Walsh CT, Rusnak F: Cyclosporin-mediated inhibition of bovine calcineurin by cyclophilins $A$ and B. Proc Natl Acad Sci U S A 1992, 89:3741-3745.

21. Zydowsky LD, Ho Sl, Baker CH, Mclntyre K, Walsh CT: Overexpression, purification, and characterization of yeast cyclophilins A and B. Protein SCi 1992, 1:961-969.

22. Fischer G, Wittmann-Liebold B, Lang K, Kiefhaber T, Schmid FX: Cyclophilin and peptidyl-prolyl cis-trans isomerase are probably identical proteins. Nature 1989, 337:476-478.

23. Gaither LA, Borawski J, Anderson L, Balabanis KA, Devay P, Joberty G, Rau C Schirle M, Bouwmeester T, Mickanin C, Zhao S, Vickers C, Lee L, Deng G, Baryza J, Fujimoto RA, Lin K, Compton T, Wiedmann B: Multiple cyclophilins involved in different cellular pathways mediate HCV replication. Virology 2010, 397:43-55

24. Ivery MT: Immunophilins: switched on protein binding domains? Med Res Rev 2000, 20:452-484.

25. Takahashi N, Hayano T, Suzuki M: Peptidyl-prolyl cis-trans isomerase is the cyclosporin A-binding protein cyclophilin. Nature 1989, 337:473-475.

26. Agresta BE, Carter CA: Cyclophilin A-induced alterations of human immunodeficiency virus type 1 CA protein in vitro. J Virol 1997, 71:6921-6927.

27. Braaten D, Franke EK, Luban J: Cyclophilin A is required for an early step in the life cycle of human immunodeficiency virus type 1 before the initiation of reverse transcription. J Virol 1996, 70:3551-3560.

28. Streblow DN, Kitabwalla M, Malkovsky M, Pauza CD: Cyclophilin a modulates processing of human immunodeficiency virus type 1 p55Gag: mechanism for antiviral effects of cyclosporin A. Virology 1998, 245:197-202.

29. De BP, Thornton GB, Luk D, Banerjee AK: Purified matrix protein of vesicular stomatitis virus blocks viral transcription in vitro. Proc Natl Acad Sci U S A 1982, 79:7137-7141.

30. Luo C, Luo H, Zheng S, Gui C, Yue L, Yu C, Sun T, He P, Chen J, Shen J, Luo $X$, Li Y, Liu H, Bai D, Shen J, Yang Y, Li F, Zuo J, Hilgenfeld R, Pei G, Chen K, Shen $\mathrm{X}$, Jiang $\mathrm{H}$ : Nucleocapsid protein of SARS coronavirus tightly binds to human cyclophilin A. Biochem Biophys Res Commun 2004, 321:557-565.

doi:10.1186/1297-9716-43-41

Cite this article as: Tanaka et al:: Suppression of feline coronavirus replication in vitro by cyclosporin A. Veterinary Research 2012 43:41.

\section{Submit your next manuscript to BioMed Central and take full advantage of:}

- Convenient online submission

- Thorough peer review

- No space constraints or color figure charges

- Immediate publication on acceptance

- Inclusion in PubMed, CAS, Scopus and Google Scholar

- Research which is freely available for redistribution 